\title{
Electrophysiology in attention-deficit/hyperactivity disorder Editorial
}

\author{
2005 International Journal of Psychophysiology, 58, 1 -3 \\ (doi:10.1016/S0167-8760(05)00212-6)
}

\begin{abstract}
Robert J. Barry, Adam R. Clarke, Stuart J. Johnstone: Brain \& Behaviour Research Institute and Department of Psychology, University of Wollongong, Northfields Avenue, Wollongong NSW 2522, Australia. Robert D. Oades: Biopsychology Group, University Clinic for Child and Adolescent Psychiatry, Virchowstr. 174, 45147 Essen, Germany
\end{abstract}

This special issue of the journal provides a sampler of current research using electrophysiological measures of activity in both the central (EEG and ERP) and the autonomic nervous systems (electrodermal), to explore the underlying deficits in attention-deficit/ hyperactivity disorder (AD/HD), with contributions from the leading research centres in the world. The focus ranges across AD/HD sufferers from childhood to adulthood, with the major DSMIV sub-types of the disorder represented, in both resting and activeprocessing conditions, and includes reports of the effects of stimulant medication. On another dimension, the papers range from a theoretical/ modeling effort, through empirical studies of various group differences, to applied clinical aspects involved in improving patient adherence to prescribed treatments.

The first two papers examine coherence, an EEG-based measure of coupling between brain regions. In the first of these, Clarke et al. examine the effects of methylphenidate on EEG coherence in boys with $A D / H D$ of the combined type. Although clear differences between the AD/HD children and controls were obtained on both inter- and intra- hemi-spheric measures, no effects of methyl-phenidate were found. The authors interpret this as suggesting that the coherence anomalies noted in AD/HD reflect differences of structure and connectivity in the brain which are not altered by stimulant medication. This has important implications for our understanding of the nature of the AD/HD dysfunction and may help shape future research. In the second coherence paper, Barry and colleagues explore a new approach to EEG coherence, adjusting observed values for the inter-electrode distance. Their study of adjusted coherence in separate samples of children with $A D / H D$ of the combined and predominantly inattentive subtypes (using DSM-IV criteria) found elevated slow-wave coherence and reduced fast- wave coherence, suggesting a complex pattern of anomalies with a frontal focus. This pattern differed little between the subtypes, but coherence was globally elevated in children with the combined type of $A D / H D$. The authors relate these effects to the different symptom patterns dominant in the subtypes, a hypothesis which may generate new focussed research.

In the first of the set of papers with an event-related potential (ERP) focus, Hermens et al. examine responses to methylphenidate in adolescents with $A D / H D$. In an initial testing session they recorded electrodermal and EEG measures from unmedicated $A D / H D$ and control groups, in a resting baseline condition and during an auditory oddball task. In a later session they retested the AD/HD children while on medication. Unmedicated 
adolescents were characterised by elevated resting EEG amplitudes in the theta band, with larger electrodermal responses and some smaller ERP component amplitudes in the oddball task. Medication impacted on both electrodermal and EEG/ERP measures in both tasks, especially in the right hemisphere, but effects were stronger in the restingstate measures.

Wild-Wall et al. report on maturation processes in brain function, using ERPs and dipole source localization. They note that the dipole sources of the mismatch negativity (MMN), an important ERP marker of automatic change detection known to document an early righthemisphere impairment of processing in children with $A D / H D$, demonstrate continued development in young adulthood. These findings are of significance for the interpretation of much of the electrophysiology of adult $A D / H D$, as they emphasise that normal developmental changes continue beyond adolescence, shifting the baseline for detection of electrophysiological anomalies.

Broyd et al. examine the effect of methylphenidate on response inhibition and the ERPs of children with AD/HD. Using an auditory cued Go/No-go task, they also recorded electrodermal activity, and unlike most studies, retested both their clinical and control groups to control for the effects of retesting when examining medication effects. Before medication, the AD/HD group had a lower $\mathrm{SCL}$ (supportive of hypoarousal in $\mathrm{AD} / \mathrm{HD}$ ), made more errors than controls, and showed ERP differences (enhanced amplitudes for N1 and P2, with reduced N2 compared with controls) suggestive of abnormal early attentional and later inhibitory processing. These differences were all reduced by methyl-phenidate. This is interpreted by the authors as reflecting a normalising of $A D / H D$ hypoarousal and inhibitory deficits with stimulant medication.

In an integrative overview, largely focussed on recent work in their laboratory, the paper by Kenemans and colleagues addresses attention, inhibition, and their underlying brain mechanisms in $A D / H D$, and the effects of methylphenidate on these mechanisms. Over-all, their data suggest that anomalies in the mechanisms involved with expectation and attention, rather than response bias or inhibitory control, underlie the impulsive behaviours of AD/HD. Assimilation of these important data by workers in the field is likely to lead to new directions in $A D / H D$ research.

The last group of papers in this special issue on $A D / H D$ turns to insights that can be obtained from measures of the restingstate EEG. In the first of these, Monastra presents a provocative report on a research effort aimed at determining and overcoming the barriers to effective treatment for this disorder. Why is it that so few diagnosed AD/HD sufferers obtain appropriate medical treatment? Monastra explores this in a sample of more than 1500 families who never began using, or had discontinued, medication prescribed for their child's AD/HD. He then implemented an intervention program which successfully overcame those barriers. His "neuro-educational" approach, including innovative uses of EEG in determining medication recommendations, and in parental education, has major clinical implications, and emphasises the practical value of using electrophysiology in the $A D / H D$ field.

The following two papers focus on heterogeneity in the EEG profiles of AD/HD children. di Michele et al. present a 
model of $A D / H D$ based on two EEGdefined subtypes identified in their previous work, and characterised, respectively, by excess alpha and excess theta activity in the resting-state EEG. Different anatomical sources of the anomalies in these frequency bands indicate that two different neural systems may be involved in AD/HD. In addition to the much-publicised consequences of hypo-function in the dopamine system in $A D / H D$, the authors put forward a role for the serotonin and noradrenaline systems. This is of interest in view of a recent general increase in the awareness of the potential contribution of these other monoamines to the wider clinical picture (see review by Oades, 2005). Exploration of the different pathways elucidated in the model should provide a rich source of hypotheses regarding the effectiveness of various medications (both stimulant and non-stimulant), and other treatments such as EEG biofeedback training, in the two EEG defined subgroups.

In the final paper, Brown et al. examine ERPs in an investigation of EEG-defined subtypes in a group of children with $A D / H D$ of the predominantly inattentive type. The EEG-derived sub-types explored by Brown et al. are labelled in terms of cortical hypoarousal and maturation lag, and do not relate directly to those of $\mathrm{di}$ Michele et al. - but may be subgroups of their excess theta group. In a mixed modality auditory/ visual oddball, the inattentive $A D / H D$ children differ from controls in terms of amplitude and latency of a number of components, generally consistent with the literature. The two EEG-defined sub-groups demonstrate a subtle difference in target N1 topography, which is broadly compatible with their labelling.

These final papers reflect efforts to accommodate the heterogeneity evident in the EEG of patients with AD/HD. Basic research clarifying the origins and implications of these differences, in a disorder which presents with such a range of symptoms spanning several separable dimensions, would seem essential for "real breakthrough" advances in explaining and understanding AD/HD.

This collection of papers presents a range of implicit and explicit views of $A D / H D$ which adds to the increasing richness of the literature on the disorder. In parallel with this, there are a number of common threads which appear through the collection.

It is clear that $A D / H D$ is universally regarded as a neurological disorder, with a range of electrophysiological anomalies which can aid in the identification, understanding, and treatment of the disorder. There are consistent resting EEG patterns, largely involving a deviant balance between fast- and slow-wave activity, with topographical differences. The coupling between brain regions in the resting state also differs in relation to both frequency and topography. Electrodermal differences, particularly lower skin conductance level, suggest hypoarousal. Such functional differences in brain state must contribute to task-related processing differences in $A D / H D$, apparent in behavioural measures of performance and atypical ERPs, with differences noted in latency and amplitude of many components. In turn these ERP differences can help clarify the processing stages involved in anomalous behavioural outputs. Complicating this picture is the observed heterogeneity of $A D / H D$. Although DSM-IV recognises different subtypes of the disorder, electrophysiology indicates even greater heterogeneity. Clarification of the implications arising from this diversity 
should be a major focus of future $A D / H D$ work in psychophysiology.

It is important to note that the techniques showcased in this volume are relevant to other streams of thought about, and approaches to, AD/HD. Concepts about $A D / H D$ in use elsewhere in child psychology, neurobiology and psychiatry, such as dysfunctional stateregulation (partly motivational and partly energetic in origin: Van der Meere, 2005) and processing reinforcement (partly motivational and partly attentional: Sonuga-Barke, 2002; Sagvolden et al., in press), have not been widely explored in Psychophysiology. The same can be said for the cognitive energetic model (Sergeant, in press) which emphasises the interplay between attention, energetics and management/executive functions. Psychophysiology needs to extend the range of topics with which it is so often involved, in order to encompass and to integrate (and test) such approaches. Too narrow a focus on the modules of information processing and response organization may make it more difficult to interpret the dysfunction in the system giving rise to $A D / H D$. Finally, the clinical implications of this collection of papers are substantial. Many of the methylphenidate effects reported here reinforce the hypoarousal interpretation of $A D / H D$ arising from the electrodermal data, and this convergence is very encouraging. It is also evident that EEG profiles may be used as an aid in deter-mining diagnosis and medication choice in AD/HD. They can be an educational device to help both patients and parents accept the existence of brain dysfunction in their case of $A D / H D$ and the need for its normalisation with medication or other prescribed treatments. This aspect of the papers presented in this special issue the demonstrated clinical utility of electrophysiology - deserves further comment and emphasis. There is often a widespread ignoring, indeed sometimes ignorance, of the potential applications of electrophysiological measures in the clinic. The editors of this collection consider that the move from laboratory to clinic is long overdue, and regard the papers presented here as strongly encouraging such a paradigm shift.

\section{References}

Oades, R.D., 2005. The roles of norepinephrine and serotonin in attention deficit hyperactivity disorder. In: Gozal, D., Molfese, D. (Eds.), Attention Deficit Hyperactivity Disorder: From Genes to Animal Models to Patients. Humana Press, New York, pp. 97- 130.

Sagvolden, T., Johansen, E.B., Aase, H., Russell, V.A., in press. A dynamic developmental theory of attentiondeficit/hyperactivity disorder (ADHD) predominantly hyperactive/impulsive and combined subtypes. Behavioral and Brain Sciences. In press.

Sergeant, J.A., Modelling attentiondeficit/hyperactivity disorder a critical appraisal of the cognitive-energetic model. Biological Psychiatry, in press.

Sonuga-Barke, E.J.S., 2002. Psychological heterogeneity in $A D / H D$ - a dual pathway model of behaviour and cognition. Behavioural Brain Research 130, 29- 36.

Van der Meere, J.J., 2005. State regulation and ADHD. In: Gozal, D., Molfese, D. (Eds.), Attention Deficit Hyperactivity Disorder: From Genes to Animal Models to Patients. Humana Press, New York, pp. 413-433. 